\title{
El desequilibrio socioeconómico consecuente de la industria de internet de las cosas ${ }^{1}$
}

\author{
The consequent socioeconomic imbalance \\ of the Internet industry of Things
}

Recibido: 31 de enero de 2016

Evaluado: 11 de abril de 2016

Aceptado: 5 de mayo de 2016

\author{
Shoumen Palit Austin Datta (Estados Unidos) \\ School of Engineering \\ Massachusetts Institute of Technology \\ shoumen@mit.edu
}

Victoria López López (España)

Universidad Complutense de Madrid

vlopez@fdi.ucm.es

\section{Resumen}

La evolución de la tecnología hacia internet de las cosas y la industria de internet está provocando cambios muy importantes en nuestra sociedad. Los cambios que se producen en las relaciones humanas así como el uso de los objetos físicos conducen a nuevas formas de comportamiento y por tanto de aprendizaje. La intimidad y la privacidad se convierten en un reto difícil de mantener y la seguridad se ve amenazada en todas sus vertientes. Sin renunciar a las ventajas del desarrollo tecnológico y a las nuevas fuentes de información en este artículo se plantea un desarrollo social responsable y consciente. Este artículo realiza una reflexión desde la evolución histórica de la tecnología industrial al desarrollo del internet de las cosas reconociendo los problemas a los que

\section{Summary}

The evolution of technology towards the Internet of Things and the Internet industry is causing major changes in our society. The changes that occur in human relationships and the use of physical objects lead to new ways of behavior and therefore of learning. The intimacy and privacy have become a difficult challenge to maintain and our security is threatened in all its aspects. Without giving up the advantages of technological development and new information sources, the present article tries to develop a responsible and conscious social development. This article makes a reflection from the historical evolution of industrial technology, to the development of the Internet of Things recognizing the problems that a new society faces and proposing as a best solution,

1 Este artículo está basado en la traducción y adaptación por parte de la doctora Victoria López del artículo titulado "Dynamic socio-economic disequilibrium catalyzed by the Internet of Things' publicado en inglés en la revista Journal of Innovation Management (ISSN 2183-0606), vol. 3, n. ${ }^{03}$, 2015; http://www.open-jim.org - HANDLE: http://hdl. handle.net/10216/80026 - Open Access: http://creativecommons.org/licenses/by/3.0 
se enfrenta la nueva sociedad y proponiendo como mejor solución la educación desde la igualdad y el respeto a los valores establecidos.

Palabras clave: internet de las cosas, desarrollo tecnológico, tecnología industrial, economía, desarrollo. the education based on equality and respect for the established values.

Keywords: Internet of Things, technological development, industrial technology, economy, development. 


\section{Introducción}

La conceptualización basada en la tecnología de internet de las cosas (IoT) y la industria de internet dio comienzo alrededor de 1988 con la obra de Mark Weiser del Centro de Investigación Xerox Palo Alto, quien sugirió que los computadores pueden "tejerse a sí mismos en el tejido de la vida diaria" e influir de forma determinante en el futuro de los negocios (Scientific American, 1991). Mark Weiser se refería al artículo seminal "El motor de vapor y el ordenador: ¿qué hace revolucionar la tecnología?", donde Herbert Simon (1987) enmarca sus pensamientos sobre la tecnología del futuro: "tienes que hacer amistad con él, hablar con él, dejar de hablar tú".

Por tanto, contrariamente a la exageración de los medios, la visión de internet de las cosas (IoT) y su significado no nacen en una presentación (Ashton, 2009) de la venta de un producto. En 2000, un primer trabajo titulado "The networked physical world" dio a luz el concepto de internet de las cosas (Manyika et al., 2011; Sarma, Brock y Ashton, 2000) y la evolución de internet industrial. Tanto el concepto como el término internet de las cosas se discutieron en un simposio en la Escuela del MIT Sloan de Administración (The MIT Sloan CIO Symposium, 2013) donde Sarma acredita Ashton para el nombre de internet of the things.

La actual conexión de los objetos del mundo físico (átomos) y la información (bits) puede llevar a otra revolución, ya predicha por muchos, como Neil Gershenfeld. Esta corriente a menudo se denomina tercera revolución industrial en relación con la primera revolución industrial y la era de la información (segunda revolución).

En algunos sectores, la tendencia actual se denomina la edad de los sistemas ciberfísicos (cyber-physical system), aunque también aparece el término industria 4.0 en referencia al mismo fenómeno.

\section{Evolución de la sociedad en el entorno tecnológico}

A lo largo de la historia, las revoluciones han surgido para remodelar el futuro y esta tercera revolución no será una excepción. Como cualquier otra revolución, generará controversia, tanto social como económica. Sin embargo, el choque del statu quo no será como hasta ahora. La sociedad ya está empezando a sufrir la colisión entre la imaginación y las ideas del viejo mundo que ahora se ven asfixiadas ante una innovación desenfrenada. La magia de la tecnología se filtra en nuestra vida cotidiana sin apenas control. Los expertos, observadores de los mercados y los actores de la industria, tienen opiniones divididas respecto de la capacidad de transformación de las tecnologías y la conectividad que necesita el internet de las cosas.

La sociedad está cambiando el formato convencional de intimidad y el concepto de privacidad se redefine y desafía los valores establecidos. La preocupación por la seguridad está muy justificada, pero todavía hay personas reticentes a renunciar a los beneficios individuales asociados a su pérdida. Los individuos claman por su seguridad y su privacidad, pero al mismo tiempo se despreocupan de estos valores cuando se les ofrece a cambio servicios gratuitos (commodities) como aplicaciones de mensajería, juegos o suscripciones a redes sociales.

En los países industrializados, es evidente la fricción financiera al surgir una fuerza de trabajo emergente de la que buena parte es trabajo no cualificado. Por otra 
parte, la automatización de procesos reduce la demanda de mano de obra no cualificada, y crea un efecto negativo en la economía y en la sociedad en su conjunto. Sin embargo, la automatización no abolirá la necesidad de mano de obra en absoluto.

Estas observaciones no son nuevas. De hecho se repiten una y otra vez desde hace siglos, aunque en diferentes tonos. De acuerdo con el economista e historiador Norman Poiré, "los cinco siglos que abarcan los años 1440 hasta 1939 fueron los más dinámicos de toda la historia”. En dicho periodo, surgieron muchos avances tecnológicos pero algunos inventos destacan por encima del resto como puntos de inflexión en la dirección de la tecnología que llevaron a cabo un cambio social decisivo. La invención de la impresión por Johannes Gutenberg en 1440 estimuló la llegada de la revolución de la información que extendió el Renacimiento en Europa. En 1609, el telescopio de Galileo Galilei marcó el comienzo de la revolución científica y la edad de la razón. La revolución industrial y el marxismo llegaron poco después de que James Watt diera a conocer su máquina de vapor en 1769. En 1939, una cuarta revolución tecnológica dio comienzo. En ese año, el doctor John Atanasoff y su estudiante graduado Clifford Berry inventaron la computadora digital electrónica y sin querer con ello la segunda revolución de la información. Un poco menos de un siglo después estamos en la cúspide de un nuevo mar de cambios, donde nos preparamos con fervor para el próximo tsunami.

La tercera revolución industrial puede estimular la gran convergencia de los sectores de la revolución industrial con la revolución de la información y otras incógnitas existentes. En el Instituto Tecnológico de Manchester, Erik Brynjolfsson y Andrew McAfee de la Sloan School of
Management del MIT (Center for Digital Business) hablan de dos enfrentamientos que pueden surgir de la tercera revolución: el aumento del desempleo y el aumento de la desigualdad (Race against the Machine y The second machine age). La visión incisiva acerca de la desigualdad también se encuentra en las obras de Joseph Stiglitz (El precio de la desigualdad) y Robert Reich (Desigualdad para todos).

Brynjolfsson y McAfee revisitan la discusión de un mayor desempleo que John Maynard Keynes describe como "desempleo tecnológico" en el 1930. Robert Frank vuelve a debatir el mismo tema en los mercados laborales en 1990 y los problemas causados por la tecnología (winner takes all) en su libro La economía Darwin.

Brynjolfsson y McAfee predicen "nuestro mundo va a prosperar en la frontera digital”, pero ¿qué pasa con la ruta de acceso a la frontera? El camino por delante está lleno de naciones enfrentadas, desnutrición, sanidad disfuncional, educación y pobreza de la energía. Tomados en conjunto, estos factores ya están alimentando fricciones socioeconómicas evidentes que pueden ser exacerbadas por los atributos necesarios para la difusión global de IoT (internet de las cosas).

Los frutos de IoT dependerán de nuestra capacidad de interoperación entre sistemas, objetos y dispositivos en diferentes ambientes así como diferentes estándares de operaciones, protocolos y aplicaciones. No cabe esperar que el mundo se esfuerce por apoyar un estándar común en un primer momento. La opinión generalizada es que las normas son una buena idea, pero son difíciles de aplicar, puesto que nadie quiere usar normativas escritas por otros. Un sistema de normalización internacional en IoT desarrollado desde la Organización Internacional de Normalización (ISO 
'International Organization for Standardization'), no parece viable a corto/medio plazo.

Por tanto, no la normalización en sí, sino la interoperabilidad entre los principales estándares puede ser la clave para la difusión de los productos y servicios de IoT y la internet industrial que alcanza en el dominio de todas las cosas mecánicas. Los líderes de la industria deben permitir estándares abiertos para interfaces (application programming interface), donde los productos de las pymes pueden conectarse a un sistema global común para acceder a la conectividad y poner sus servicios de valor añadido, motores de análisis o mejorar aplicaciones. El despliegue sistemático de datos abiertos (open data) y su conectividad es fundamental para la adquisición de datos y la difusión de IoT (López, 2014, 2015).

En última instancia, la capacidad inteligente a partir de la extracción de datos impulsará la propuesta de valor de la conectividad. La economía de los costes de transacción (naturaleza de la firma de Ronald Coase, 1937) determinará el retorno de la inversión que puede influenciar la adopción por negocios y empresas.

Los procedimientos tradicionales de retorno a corto plazo, y muy frecuentes en el mundo de los negocios, pueden retardar la adquisición de datos. Una inversión inadecuada puede limitar las herramientas necesarias para acumular una masa crítica de datos. Así, sin datos suficientes, las herramientas analíticas pueden fallar en el descubrimiento de patrones ocultos en los datos. Sin embargo, los procesos de descubrimiento de patrones son muy necesarios especialmente cuando los motores de análisis dinámicos en tiempo real son un camino a la monetización de IoT. En este aspecto, se pueden crear nuevas fuentes de ingresos a partir de micropagos basados en el modelo de pago por análisis de arbitraje de la información (pay-per-analytics model), que utiliza análisis predictivo inteligente para mejorar el apoyo a la toma de decisiones semiautónoma.

Una lección de adquisición y análisis de datos puede extraerse del experimento clásico de la mecánica cuántica, que describe el experimento de la doble rendija de Young. Una variación de este experimento se realizó en el Hitachi Laboratorio Central de Investigación (HITACHI) en Kokubunji por el doctor Akira Tonomura (1942-2012). Este segundo experimento (HITACHI, 2015) reveló la existencia de un patrón de interferencia a partir de electrones individuales, pero no se observó hasta que se permitió el paso de un número de electrones suficientemente grande a través de la rendija. La lectura de este experimento para los negocios es obvia, porque la ejecución de experimentos a pequeña escala puede no ofrecer resultados adecuados e incluso podría proporcionar indicaciones equivocadas (puesto que no se puede construir un elefante tomando un ratón como modelo). En este sentido, este trabajo es intuitivo, porque sugiere despliegues a gran escala como clave para extraer el valor de los datos y la importancia de las herramientas y las tecnologías que, cuando se combinan y convergen, pueden aportar excelentes soluciones.

En la historia de los procesos tecnológicos, se pueden encontrar ejemplos como las estrategias que permitieron la difusión de la electricidad (The economic future in historical perspective, editado por P. A. David y M. Thomas, Oxford University Press, 2003). En el trabajo The innovators dilemma, de Clayton Christensen, el término disruptive es una exageración basada en el concepto original de las tecnologías de procesos generales (general process technologies) introducidas durante la era de la 
electrificación para indicar integración sistémica frente al uso ad hoc del término slap-on. Christensen hizo un mal uso del témino disruptive así como un erróneo análisis de datos que condujo a concluir que todo es perjudicial (Andrew King, MIT Sloan School of Management).

\section{Cambios en la organización}

Durante los últimos quince años se ha observado una falta de integración sistémica de etiquetas RFID (radio frequency identification) de identificación por radiofrecuencia, muy habituales en todo tipo de establecimientos para identificar los productos. A pesar de su uso muy extendido, la industria no se ha beneficiado suficientemente de la capacidad de las etiquetas RFID para adquirir grandes volúmenes de datos desde un enfoque de sistemas. Como resultado, es posible que hayamos fracasado para ofrecer una adecuada transparencia en las cadenas de suministro y el ahorro del valor de la cadena se mantiene muy por debajo de lo previsto. En WalMart (Is RFID dead? Florian Michahelles [2010] Auto-ID Labs St. Gallen, ETH Zurich), un trabajo sobre el abandono de la iniciativa de las tarjetas RFID, se sugiere que no es un fracaso de la tecnología, sino un uso inadecuado de la herramienta de datos en el contexto del proceso de negocio. Es un fracaso de cambio organizacional.

Internet de las cosas puede aprender de la historia de la electrificación y la tecnología por radiofrecuencia RFID, con el fin de encontrar mejores maneras de penetrar progresivamente nuestra realidad cotidiana a través de sistemas de integración, conectividad y aplicaciones. Internet de las cosas debe evolucionar a internet de los sistemas (IoS). La conectividad de sistemas en nuevos ecosistemas puede generar el siguiente tsunami de rentabilidad. A su vez, aumentarán aún más los problemas de seguridad, privacidad, confianza y ética relacionados con nuestros programas de política social. La conectividad y las comunicaciones con los objetos y procesos cambiarán nuestra forma de interactuar y nuestro comportamiento tanto en la vida personal como en el entorno profesional en la era de la internet de los sistemas (IoS).

\section{Nuevos modelos de negocio}

La búsqueda de la autonomía en el cuidado de la salud, el transporte y la fabricación creará nuevas soluciones y germinará con nuevos modelos de negocio. La predicción de enfermedades como el cáncer con una década de anticipación no es una ilusión. Los vehículos con sistemas autónomos de estacionamiento, los camiones de carga y la distribución de paquetería sin interacción humana son ya noticias del pasado. La muerte del inventario y el nacimiento de la fabricación y distribución por demanda (dMODE) es el embrión de la Manufacturación 5.0 con el desarrollo de los sistemas de impresión $3 \mathrm{D}$. Con el desarrollo de válvulas cardiacas, nanosatélites, la medición de la humedad del suelo por la NASA (SMAP) para la agricultura de precisión, la fabricación de agua potable desalinizada libre de arsénico y otros avances tecnológicos (neurosynaptic web y neuromorphic chips), podemos decir que ya ha comenzado el próximo viaje de cien años.

Según Jeff Immelt, CEO de General Electric,

En el futuro se espera un tejido abierto y global de máquinas altamente inteligentes que se conectan, se comunican y 
colaboran con nosotros. La internet industrial no trata de un mundo dirigido por los robots, sino de combinar el mundo con mejores tecnologías para resolver nuestros mayores retos. Se trata de crear un mundo económicamente y ambientalmente sostenible, se trata de curar las enfermedades ahora incurables, y de preparar nuestras infraestructuras y ciudades durante los próximos 100 años.

La fricción económica es evidente tras la automatización de tareas repetitivas que hasta ahora han supuesto puestos de trabajo de ingresos medios: el personal de los bancos, el personal que atiende las ventanillas e incluso los profesores de cualquier grado en el sistema educativo (K-16 Teachers) están siendo sustituidos por cajeros automáticos, quioscos de autoservicio y los cursos abiertos masivos (MOOC). Esto no solo se debe a internet de los sistemas (IoS), sino también a la integración de la computación en nuestra vida cotidiana, según lo predicho por Herbert Simon en 1987 y popularizado por Mark Weiser en 1991.

\section{Internet de los sistemas (IoT)}

La conectividad de internet de los sistemas (IoS) con una sección transversal mayor de los objetos y procesos además de la exposición a mayor grado de supervisión (por ejemplo en cuidado de la salud) inducirá cambios en el comportamiento con el aumento de la difusión de internet de las cosas. La evolución de la actividad racional frente a la irracional debe ser observada y analizada (Thinking fast and slow, Daniel Kahneman). El resultado de estos análisis debe tenerse en cuenta al diseñar futuros productos y servicios, por ejemplo, hospitales wireless del futuro o máquinas de resonancia magnética con repostaje automático de hidrógeno o máquinas de rayos $\mathrm{X}$ portátiles para ser usadas en las chozas en la Amazonia. La utilidad de estos avances puede depender de los valores socioeconómicos (Scarcity, Sendhil Mullainathan) y de la etapa de desarrollo del país o la región donde se aplica (Development as freedom, Amartya Sen).

La predicción de que la conectividad va a cambiar el comportamiento de las personas tiene sus raíces en el principio fundamental de la física de partículas. El "efecto del observador", como se le llama, se refiere a los cambios que el acto de la observación produce cuando se observan fenómenos (y que no debe confundirse con el principio de incertidumbre propuesto por Werner Heisenberg). El primero puede explicar por qué cantamos en la ducha, pero no en público.

Cabe señalar que el comportamiento combinado de hardware y software integrados con objetos físicos cambiará si alguno de los componentes cambia, incluso si se sustituye por otro prácticamente idéntico. Además de los cambios en el comportamiento, el reequilibrio económico será lento, porque son necesarios cambios sustanciales en nuestro sistema de educación para optimizar el consumo social de los frutos de la tecnología. Ninguna cantidad de cursos de tecnología o cursos en línea evitará la propagación de la ruptura en nuestro tejido económico a menos que reformemos la educación pública y restauremos el respeto a las autoridades educativas, nos centremos en rejuvenecer todos los aspectos de la investigación científica, en restablecer la dignidad a los maestros y volver a encender la pasión que se espera de un profesor. 
La cadena de suministro emergente de talento debe incluir la gran cantidad de niñas que sobresalen en matemáticas y que pueden programar y escribir coherentemente. Es esencial que las mujeres persigan el nivel más alto de la ciencia, la ingeniería, las matemáticas, la economía y la filosofía. ¿Cómo podemos aceptar que alrededor de $50 \%$ de la energía del cerebro se quede fuera de la mano de obra especializada?

Las mujeres educadas ayudarán a educar niñas y niños respetuosos y dignos. El trabajo conjunto acelerará la innovación masiva en paralelo de las lejanas grietas del mundo en el que ya se está marcando el comienzo de movimientos tectónicos, incluso en las tareas más tradicionales. El enfoque de análisis-parálisis de los gigantes puede conducir a su extinción si siguen siendo ajenos al hecho de que el fracaso es el nuevo camino hacia el éxito, el fracaso es la nueva clave para el éxito y el fracaso como mantra para el éxito de la economía emergente.

La distribución de la innovación exige un enfoque empresarial y un salto a múltiples niveles en lugar de soluciones mágicas (Innovation: The attacker's advantage, Richard Foster). La industria del taxi frente a servicios como UBER (www.uber.com), la industria hotelera frente a aplicaciones como Airbnb (www.airbnb.com) y las agencias de trabajo temporal frente a oDesk (www.upwork.com) son buenos ejemplos. La explosión de herramientas de ingeniería ha reducido drásticamente el tiempo necesario para introducir el ciclo de innovación por lo que ha comprimido enormemente el tiempo desde la concepción (desarrollo de la cadena de suministro) a la realización (cumplimiento de la cadena de suministro). Los gigantes de la industria deben aprovechar esta explosión y facilitar la creación de plataformas de código abierto donde concurran las ideas y el desarrollo inteligente de aplicaciones. El flujo de microingresos de miles de millones de clics en la web de un producto será el elemento diferenciador y el servicio de datos de valor ańadido estará relacionado con análisis inteligente de datos y la entrega de información útil antes que los datos perezcan. La inteligencia de negocio es el factor clave en la economía resultante.

Sin embargo, el carácter perecedero de los datos cambia cuando la acumulación de datos en el tiempo es mucho más crítica para un análisis predictivo (por ejemplo en medicina, en la evolución de algunas enfermedades) y no de datos con corta vida media (por ejemplo el tiempo medio entre fallos MTBF [mean time between failures] o la métrica de piezas en una turbina). El transporte y almacenamiento de datos son importantes, pero los consumidores pueden estar dispuestos a pagar solo por su análisis en tiempo real y los resultados de aplicar las técnicas inteligentes. Los consumidores esperan que los datos en bruto sean abiertos y libres (gratis).

Aun así, todas estas ventajas son temporales. La sabiduría financiera de microingresos de arrendamiento de las plataformas es una de las razones por las que Apple abrió su bus para que cualquiera puede subir sus aplicaciones. Las aplicaciones llegan de todas partes del mundo como una avalancha económica y le permite a Apple, como propietaria del canal, agregar micropagos mediante la innovación abierta. Small data de millones de fuentes es la razón por la que Apple sonríe con la base de datos más grande del mundo de pagos, con una suma de 99 centavos de dólar en cada pago. El éxito de PayPal se ve alimentado por firmas como Tesla, que es capaz de regalar el coche que fabrica para vender baterías intercambiables a base de grafeno y servicios a 
bordo (utilizando software definido en red, software defined networking). El futuro del automóvil Apple podría usar la red eléctrica móvil en lugar de la red inmóvil inteligente para distribución de energía off-grid. Los productos gratuitos con servicios basados en pago por el uso (pay per use) son ya una estrategia de negocio madura (impresoras frente a compra de tinta, teléfonos móviles frente a servicios, cafeteras frente a cápsulas de café) para ampliar las microganancias y garantizar una larga vida a los productos. Rolls Royce venderá horas de uso (thrust hours) en lugar de motores de avión; Google venderá planes de ahorro de energía solar (solar savings plans) en lugar de alertas y Bosch no venderá sensores físicos sino la inteligencia de los sistemas de sensores.

Se espera que la propagación de IoT y IoS dé lugar a nuevos productos y servicios (Datta, 2015b). El consumo de estos bienes y las mejoras en la eficiencia puede generar una magnitud de crecimiento económico que está induciendo mucha euforia en los CEO (chief executive officer) de las empresas. Según General Electric, Cisco y otras compañías, IoT y la internet industrial (IIoT) pueden sumar entre US\$14 000 millones y US\$19 000 millones a la economía global en la próxima década. Para que estas cifras se materialicen, debe ocurrir una explosión en el consumo. Los cientos de millones de personas que están escribiendo en las redes o publicando fotografías para mantener a flote la burbuja de la capitalización del mercado de los medios sociales pertenecen a un grupo social con ingresos insuficientes para pagar coches que hablan o una laparoscopia robótica. El consumidor educado es el mejor cliente. La burbuja económica de Twitter como medio social puede estar limitada por la cantidad de "energía bajo la curva" y la exuberancia irracional puede alimentar la próxima recesión global que podría estar a la vuelta de la esquina (20202022). Con 2008 como el año de la última recesión, se espera la próxima alrededor de 2022 si el "ciclo de auge y caída" todavía exhibe la periodicidad de catorce años, como explica Finn Kydland (Premio Nobel de Economía 2004).

\section{Reflexiones finales}

En los Estados Unidos están surgiendo varias iniciativas en la industria y el mundo académico para hacer frente a la próxima generación de avances en el espacio de IoT, IIoT, IoS y las interesantes posibilidades de investigación en sistemas ciberfísicos. En el periodo 2014-2015, se formaron varios consorcios de líderes del mercado. En investigación, varios grupos académicos están liderando el camino con nuevos inventos e innovación. También la Unión Europea ha financiado un programa de varios años denominado Horizonte 2020 por una suma de más de US\$100 000 millones para explorar el crecimiento de IoT y ayudar a la cosecha de la bonanza económica asociada.

Pero será un error atroz por parte de los líderes globales cegarse por las proyecciones económicas sin prestar atención a un ajuste necesario del motor de la educación. ¿Una smartcity puede ser realmente inteligente sin ciudadanos inteligentes (Datta, 2015a)? ¿Serán las smartcity también ciudades sostenibles (López, 2014, 2015; López et al., 2015)?

La humanidad necesita soñadores (Datta, 2009) y la educación (Datta, 2014, 2014b) es la quinta esencia (Salt: A world history, Mark Kurlansky) que actúa como el proveedor de inspiración, imaginación, invención, innovación y unidades de implementación de las ideas (Datta, 2015b). La educación de un niño puede cambiar el 
destino de un hombre. La educación de una niña puede cambiar el destino de una nación.

\section{Referencias}

Ashton, K. (2009). That 'internet of things' thing. RFiD Journal, 22(7), 97-114.

Datta, S. (2014a). Far reaching changes in the near future. Recuperado de

http://bit.ly/Book-by-S-Datta

Datta, S. (2014b). Internet of Systems (IoS) - Economic Re-equilibration Catalyzed by Internet of Things (IoT). Recuperado de http://dspace.mit.edu/handle/1721.1/86935

Datta, S. (2015a). Smart cities. Recuperado de http://bit.ly/SMART-CT

Datta, S. (2015b). Presentation of Datta 2015. Recuperado de http://bit.ly/RE-VIEW-IOT

Datta, S. (2013). Humanity Needs Dreamers. Recuperado de http://bit.ly/HND-250

Foster, R. N. (1986). Innovation: The attacker's advantage. Nueva York: Summit.

HITACHI (2015). Quantum measurement. Recuperado de http://www.hitachi.com/rd/portal/highlight/quantum/ index.html\#anc0 4

Kahneman, D. Pensar rápido, pensar despacio, Ed. Farrar, Straus and Giroux, Nueva York, 2011.

López, V. (2014). Big and open data: Challenges for Smartcity. Recuperado de http:// www.slideshare.net/vlopezlo/big-open-data-challenges-for-smartcity.
López, V. (2015). Open Data para Smartcity. Recuperado de https://www.youtube.com/ watch?v=x9G_1-0rLIE

Lopez, V., Miýana, G., Sýnchez, O., Gonzýlez, B., Valverde, G. y Caro, R. (2015). Big+Open Data: some applications for a Smartcity. En Progress in Informatics and Computing (PIC), 2015 IEEE International Conference on (pp. 384-389). IEEE.

Manyika,J., Chui, M., Brown, B., Bughin, J., Dobbs, R., Roxburgh, C.,\& Hung Byers, A.(2011). Big data: The next frontier for innovation, competition, and

productivity. Recuperado de http://tinyurl. $\mathrm{com} /$ industrial-internet

Sarma, S., Brock, D. L. y Ashton, K. (2000). The networked physical world. Recuperado de http://cocoa.ethz.ch/downloads/2014/06/ None_MIT-AUTOID-WH-001.pdf

Shah, A. K., Shafir, E. y Mullainathan, S. (2015). Scarcity frames value. Psychological Science, 7(1), 1-36.

Stiglitz, J. E. (2012). El precio de la desigualdad. Madrid: Taurus.

The MIT Sloan CIO Symposium (2010). 2010-09 The internet of Things. Recuperado de https://www.youtube.com/ watch?v=44MLERLwxig

h t t p s: / / www.you tube.com / watch? $v=44$ MLERLwxig

Weiser, M. (1991). The Computer for the 21st Century-Scientific American Special Issue on Communications. Computers, and Networks, 265(3). 